\title{
Approaches for Establishing Clinically Relevant Dissolution Specifications for Immediate Release Solid Oral Dosage Forms
}

\author{
Andre Hermans, ${ }^{1}$ Andreas M. Abend, ${ }^{1,10}$ Filippos Kesisoglou, ${ }^{1}$ Talia Flanagan, ${ }^{2}$ Michael J. Cohen, ${ }^{3}$ \\ Dorys A. Diaz, ${ }^{3}$ Y. Mao, ${ }^{1}$ Limin Zhang, ${ }^{4}$ Gregory K. Webster, ${ }^{5}$ Yiqing Lin, ${ }^{6}$ David A. Hahn, ${ }^{7}$ \\ Carrie A. Coutant, ${ }^{8}$ and Haiyan Grady
}

Received 3 February 2017; accepted 16 June 2017; published online 22 August 2017

\begin{abstract}
This manuscript represents the perspective of the Dissolution Analytical Working Group of the IQ Consortium. The intent of this manuscript is to highlight the challenges of, and to provide a recommendation on, the development of clinically relevant dissolution specifications (CRS) for immediate release (IR) solid oral dosage forms. A roadmap toward the development of CRS for IR products containing active ingredients with a non-narrow therapeutic window is discussed, within the context of mechanistic dissolution understanding, supported by in-human pharmacokinetic (PK) data. Two case studies present potential outcomes of following the CRS roadmap and setting dissolution specifications. These cases reveal some benefits and challenges of pursuing CRS with additional PK data, in light of current regulatory positions, including that of the US Food and Drug Administration (FDA), who generally favor this approach, but with the understanding that both industry and regulatory agency perspectives are still evolving in this relatively new field. The CRS roadmap discussed in this manuscript also describes a way to develop clinically relevant dissolution specifications based primarily on dissolution data for batches used in pivotal clinical studies, acknowledging that not all IR product development efforts need to be supported by additional PK studies, albeit with the associated risk of potentially unnecessarily tight manufacturing controls. Recommendations are provided on what stages during the life cycle investment into in vivo studies may be valuable. Finally, the opportunities for CRS within the context of post-approval changes, Modeling and Simulation $(\mathrm{M} \& S)$, and the application of biowaivers, are briefly discussed.
\end{abstract}

KEY WORDS: BCS; biowaivers; clinically relevant dissolution specifications; PBPK modeling; SUPAC.

\footnotetext{
This article was developed with the support of the International Consortium for Innovation and Quality in Pharmaceutical Development (IQ, www.iqconsortium.org). IQ is a not-for-profit organization of pharmaceutical and biotechnology companies with a mission of advancing science and technology to augment the capability of member companies to develop transformational solutions that benefit patients, regulators, and the broader research and development community.

${ }^{1}$ Pharmaceutical Sciences and Clinical Supply, Merck \& Co., Inc., West Point, Pennsylvania 19486, USA.

${ }^{2}$ Pharmaceutical Technology and Development, AstraZeneca R\&D, Macclesfield, Cheshire, UK.

${ }^{3}$ Worldwide Research and Development, Global Chemistry and Manufacturing Controls, Pfizer Inc., Eastern Point Road, Groton, Connecticut 06340, USA.

${ }^{4}$ Drug Product Science and Technology, Bristol-Myers Squibb Company, New Brunswick, New Jersey 08903, USA.

${ }^{5}$ Global Pharmaceutical Research and Development, AbbVie Inc., North Chicago, Illinois 60064, USA.

${ }^{6}$ Analytical Development, Biogen Inc., Cambridge, Massachusetts 02142, USA.
} 


\section{PURPOSE}

The purpose of this paper is to describe a roadmap for the development and leverage of clinically relevant dissolution specifications (CRS) for immediate release oral solid dosage forms of non-narrow therapeutic window drugs. There are three objectives for presenting this roadmap: (1) to describe multiple approaches for establishing clinical relevance for dissolution methodology, (2) to outline how to leverage clinically relevant dissolution methodology and specifications for the confirmation of the final drug product quality, and (3) to suggest recommendations on supporting scale-up and post-approval changes using the established CRS.

Historically, dissolution specifications have been set by controlling the formulation and process within precedented conditions, and setting the method conditions and acceptance criteria (=specification) according to current guidance (1).

While this approach is still acceptable, recent advancements in technology and modeling and simulation significantly contribute to enhanced product understanding. Nevertheless, this enhanced product knowledge has historically been underutilized in setting specifications for product approval and in support of post-approval changes. Through this approach, the dissolution specification should not only confirm adequate process control but also imply consistent in vivo performance with respect to efficacy and safety.

In order to address this gap, we propose a roadmap as described in this article.

\footnotetext{
${ }^{7}$ Genentech, Inc., 1 DNA Way, South San Francisco, California 94080, USA.

${ }^{8}$ Small Molecule Design \& Development, Eli Lilly \& Co., Lilly Corporate Center, Indianapolis, Indiana 46285, USA.

${ }^{9}$ Takeda Development Center Americas, Inc., One Takeda Parkway, Deerfield, Illinois 60015, USA.

${ }^{10}$ To whom correspondence should be addressed. (e-mail: andreas_abend@merck.com)
}

Abbreviations: API Active Pharmaceutical Ingredient, AUC Area under Curve, BCS Biopharmaceutical Classification System, BioRAM Biopharmaceutics Risk Assessment Roadmap, $\mathrm{C}_{\max }$ Maximal concentration, CMA Critical Materials Attribute, CPP Critical Process Parameter, CQA Critical Quality Attribute, CRS Clinically Relevant Specifications, EMA European Medicines Agency, FDA Food and Drug Administration, FIP International Pharmaceutical Federation, ICH International Council for Harmonization of Technical Requirements for Pharmaceuticals for Human Use, IR Immediate Release, IVIVC/R In-vitro-in-vivo correlation/ relationship, M\&S Modeling and Simulation, MR Modified Release, SUPAC Scale up and Post-Approval Changes, PBPK Model Physiologically Based Pharmacokinetic Model, PD Pharmacodynamic, PK Pharmacokinetic, PSD Particle Size Distribution, QC Quality Control, QbD Quality by Design, QRA Quality Risk Assessment, QTPP Quality Target Product Profile, XR Extended Release

\section{BACKGROUND}

Since the publication of Janet Woodcock's final report on pharmaceutical quality for the twenty-first century in 2004 (2), pharmaceutical companies have embraced Quality by Design (QbD) concepts in drug development. Guidance and expectations are given in regulatory documents including ICH Q8, Q9, and Q10 (3-5). In addition, several published articles, conference talks, and podium discussions are available that discuss this concept at length (6-8). QbD is a prospective approach that provides assurance of robust manufacture of reliable product quality for patients.

Patients, health care providers, and payers expect medicines to demonstrate consistent product performance, i.e., safety, quality, and efficacy. A product's critical quality attributes (CQAs), which are understood during product development, ultimately delineate the boundaries of the product control strategy in a registration market application. A product control strategy under an enhanced (QbD) approach is represented by the functional relationship between CQAs and the critical process parameters and critical material attributes described in the drug substance (also referred to as active pharmaceutical ingredient) and drug product manufacturing processes. Drug substance and drug product specifications provide confirmation of appropriate process control and consistent product quality. However, a product control strategy may not assure consistent safety and efficacy unless drug product specifications are clinically relevant.

Clinical relevance requires establishing in vitro acceptance criteria for dissolution that control against changes in product attributes that could influence in vivo performance as part of the control strategy. This relationship is most simply built by alignment of acceptance criteria against testing results obtained on materials used in pivotal safety and efficacy clinical trials. For several solid oral dosage forms, in vitro dissolution or drug release criteria provide a connection to, and serve as a predictor of, in vivo performance. However, this empirical relationship may be imprecise depending on, e.g., the Biopharmaceutics Classification System (BCS) classification of the drug. Recent communications from a regulatory perspective have questioned the clinical relevance of these specifications, and the topic has been the subject of several publications and presentations from both regulatory agencies and industry (9-15).

In vitro dissolution testing is used throughout drug product development to guide formulation process development and to control product quality. The goal of the dissolution evaluation is to probe the in vitro rate and extent of drug solubilization to inform selection of formulation candidates or the impact of process conditions on potential in vivo performance. At earlier stages of product development, dissolution experiments under biorelevant conditions are often applied to rank order formulations for further development or to evaluate if formulation and process variations could lead to differences in bioperformance. The hypothesis at this stage of product development is that in vitro differences will be reflective of in vivo performance. This assumption is continuously tested throughout drug development as more data are generated that allow for refinement of the dissolution method. However, the link between dissolution and in vivo performance is often still theoretic or derived from data from a limited number of batches used in clinical studies. It is often difficult to assess whether an observed 
change in dissolution profile will have a significant impact in vivo. For IR drug products containing highly soluble drug substances, a single-point dissolution acceptance criterion for suitably complete release within $60 \mathrm{~min}$ is typically expected. IR tablets formulated with poorly soluble active pharmaceutical ingredients (APIs) however often require the addition of surfactants to achieve complete release within 60 min which may not be reflective of the intraluminal conditions the drug will experience with oral dosing. In both instances (highly and poorly soluble drugs), a globally acceptable dissolution method will likely be capable to detect significant process or formulation changes but may not be able to correlate these with potential bioperformance changes.

To enhance the understanding of the relevance of dissolution acceptance criteria to in vivo performance of a drug product, additional investigational work may sometimes be needed. For example, identifying which steps in the manufacturing process are expected to have the most significant impact on the in vivo release rate of the drug product may be essential in developing an appropriate in vitro dissolution method to guide drug product development. The actual capability of such a method to distinguish between batches that meet the desired in vivo performance needs in vivo investigation to determine what dissolution changes are significant.

In the past, both industry and regulators acknowledged that dissolution product quality "sameness" can be demonstrated by showing similarity factor $f_{2}>50$ (SUPAC) $(16,17)$, but neither sameness nor nonsameness may not be clinically relevant, unless the dissolution test is clinically relevant. Thus, using statistical tools (e.g., $f_{2}$ ) to evaluate sameness of product dissolution made before and after process and/or formulation changes may only be meaningful if the method(s) used to monitor the impact of these changes in vitro is appropriate to detect relevant changes of in vivo exposure. This is a key driver for developing clinically relevant dissolution tests.

For IR products formulated with a highly soluble drug substance (Biopharmaceutical Classification System 1 and 3 compounds), a criterion of $\geq 85 \%$ dissolved in 30 or $15 \mathrm{~min}$, respectively, is proposed to be acceptable to demonstrate bioequivalence as suggested by the recent draft FDA dissolution guidance (18). For other immediate release solid oral products, the in vivo relevance of dissolution changes can be addressed by studying the PK behavior of product made under different compositional or process conditions, so-called formulation or process variants, and developing dissolution methods that are sensitive to changes that are significant. If a PK response is seen for the different product variants, ideally the next step is to link the dissolution data and the observed PK response. This may be achieved either with an in vitro/in vivo correlation (IVIVC) that may be established with traditional deconvolution/convolution approaches or a physiologically based oral absorption/pharmacokinetic model (referred to as oral absorption/PBPK models for the rest of this document). This manuscript describes science-based approaches for establishing clinically relevant specifications for immediate release products with a non-narrow therapeutic window. Some current industry practices for these approaches are presented and discussed within the context of regulatory opinions expressed either during product application reviews or in public meetings, publications, etc.

Linking in vitro and in vivo (PK) data with formulation and process variants to establish dissolution method specifications and to demonstrate that a product will retain its desired bioperformance after formulation or process changes may present practical challenges in some cases. For instance, drug product developers may find themselves in situations wherein performing a standalone study to explore the impact of process variants on in vivo performance may be difficult or even unethical. Examples include highly potent compounds that cannot be dosed to healthy volunteers, such as many small molecule oncology drugs, and controlled substances. In addition, for some drug products such as those of orphan drugs or accelerated development programs, the investment in making multiple product variants and performing several relative bioavailability studies to support CRS may not be practical. In these situations, alternative approaches to ensure that the proposed dissolution method and specification can assure suitable in vivo performance will be needed, and may rely more heavily on in silico models (typically physiologically based pharmacokinetic models) as well as the traditional use of dissolution data from materials used during safety and efficacy studies performed in the clinical program. It is also worth noting that there may be opportunities for understanding formulation behavior (and thus refine dissolution methods) by leveraging data from other clinical studies that are conducted during drug development where different formulation presentations may be employed. For example, it is not uncommon that first-in-human (FIH) studies are conducted with a formulation that differs from the formulation which will be developed for further clinical development. To determine the most appropriate formulation and to support the commercial product, different formulation variants may be tested in relative bioavailability studies which may not be dedicated IVIVC studies (19).

\section{CLINICALLY RELEVANT SPECIFICATION ROADMAP}

"Clinically relevant dissolution specification" implies that the in vitro dissolution method confirms acceptable in vivo performance of the product. A biorelevant dissolution method, which by a definition suggested by the FIP is performed in aqueous media resembling the intraluminal environment the drug product passes through during administration, can also be clinically relevant. In contrast, a clinically relevant dissolution method can be performed in "non-biorelevant" dissolution media as long as the method and acceptance criteria are capable of confirming or predicting in vivo performance of the drug (20-22). Every drug product manufacturing process is subject to variability, and it is expected that a clinically relevant dissolution method is capable of discriminating if these variations have, or do not have, an impact on product in vivo performance. The sources of this variability may stem from differences in raw materials, including the drug substance and excipients, differences in processing conditions, and even changes in the day-to-day environmental conditions. Within the context of thorough 
product development, such variability can be evaluated for significance.

It can be challenging to judge if this variability has an impact on product bioperformance by solely relying on a dissolution method and acceptance criteria without establishing an understanding of the in vivo (in-human) relevance of the method, or, alternatively, without establishing tight acceptance criteria to assure similarity. Therefore, understanding the link of dissolution performance to pharmacokinetic performance can be a key factor in developing product understanding and can play a major part of the development strategy and product life cycle management.

The approaches described below on how to establish clinically relevant dissolution specifications for immediate release solid oral dosage forms are based on the interpretation of meeting materials and publications issued by scientists working for regulatory agencies and in the pharmaceutical industry (9-15).

During development, drug products should be designed with the patient in mind, wherein the Target Product Profile (TPP) delineates attributes of the product to be delivered to meet patient safety, efficacy, quality, and suitability for administration. The Quality Target Product Profile (QTPP) identifies the specific product Critical Quality Attributes (CQAs) required to deliver the desired TPP. How to achieve these quality characteristics is evaluated during formulation and process development. Aspects that may significantly impact in vivo performance are often assessed by in vitro dissolution. Therefore, developing an enhanced understanding of the dissolution mechanism and how formulation and process variables influence dissolution is fundamental for developing clinically relevant criteria.

The role of dissolution testing, and the test employed, may change during development of the product. Early in development, a variety of explorative approaches (i.e., dissolution in biorelevant media (23), multi-stage dissolution (24,25), biphasic/multicompartmental (gastro-intestinal models) (26), dissolution in non-sink conditions, etc.) or dissolution in multi-pH media can be used to evaluate the possible impact of formulation and process factors on the in vivo performance of the dosage form. In early development, data from these methods along with other physicochemical information, such as permeability, can be combined with physiologically based oral absorption/pharmacokinetic modeling to predict formulation performance in anticipation of clinical studies. These models can be further refined as more data become available. Application of such models can significantly enhance formulation and process development and the assessment of biopharmaceutics risk factors. The use of this type of physiologically based pharmacokinetic (PBPK) model has been a subject of several recent publications $(27,28)$.

Understanding the dissolution mechanism of a drug product and identifying which aspects of the formulation and manufacturing process have the most significant impact on the in vitro release rate are critical in developing a dissolution method. Figure 1 illustrates the mechanistic steps involved when a typical solid oral dosage form dissolves. The dissolution process can be divided into three steps: tablet erosion/disintegration into granule particles, granule disintegration into primary drug particles, and API dissolution. Potential CQAs, potential Critical Process Parameters (CPPs), and potential Critical Material Attributes (CMAs) are listed for each of the three mechanistic steps. These are typically the focus of evaluation in development, and build the foundation of enhanced product understanding.

In early drug product development, where different formulations and processes are considered, changes in both composition and process can impact each of these dissolution events differently. For a given formulation and manufacturing process, compositional and process variants may affect one of these dissolution steps more strongly than the others. Experimentation such as disintegration, intrinsic API dissolution, and granule dissolution can help in understanding the relative contribution of each step to the overall dissolution rate.

If the rates of the mechanistic steps shown in Fig. 1 are very different, then the slowest step determines the overall dissolution rate. In this case, the effect of variations in formulation and process factors likely to impact this slowest dissolution step should be most closely evaluated. CPPs and CMAs can be identified through this process.

As part of selecting the QC dissolution method for the product, one is often faced with the challenge of valuing and evaluating the discriminating power of the method. In order to address this challenge, one can perhaps study the impact of the aforementioned CPPs and/or CMAs in clinical studies, or one

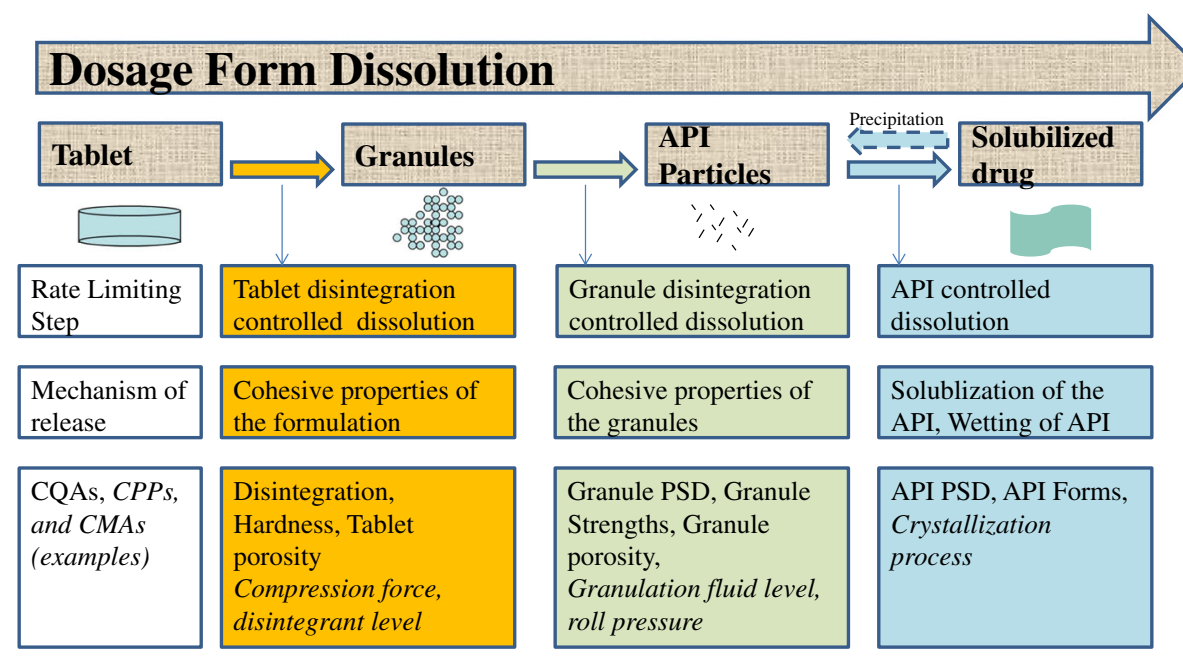

Fig. 1. Typical dissolution mechanism and factors to explore when developing IR solid oral dosage forms 
might accept tight specifications and mitigate potential biopharmaceutical risk by setting tight manufacturing controls.

When performing these development activities, the BCS classification system can aid in estimating if process/formulation variations and changes in product dissolution are likely to have an impact on in vivo performance (29). The recent draft FDA dissolution guidance (18) suggests that immediate release solid oral dosage forms comprising highly soluble compounds, which achieve $\geq 85 \%$ dissolved in $500 \mathrm{~mL}$ of $0.01 \mathrm{~N} \mathrm{HCl}$ with gentle agitation in either $30 \mathrm{~min}$ (BCS class 1) or $15 \mathrm{~min}$ (BCS class 3), provide assurance that they will deliver similar bioperformance. In other words, a Q of $80 \%$ (i.e., $\geq 85 \%$ dissolved) in $15 \mathrm{~min}$ (BCS class 3) or $30 \mathrm{~min}$ (BCS class 1) should be considered clinically relevant.

In all other cases, such as BCS classes 2 and 4 or BCS classes 1 and 3 that do not meet the aforementioned dissolution criteria, applying the $f_{2}$ similarity factor vs. lots used in pivotal clinical studies can be a useful baseline, although likely conservative, approach to mitigate biopharmaceutical risk if the method is appropriately discriminating.

To extend understanding, IVIVC/Rs and/or oral absorption/PBPK models in conjunction with data from preclinical (animal) models can also be used to probe the impact of changes in the dissolution profile (e.g., resulting from formulation and process changes) on the PK profile. The results from these simulations can then be used to refine understanding of the biopharmaceutical risk. It is also worth emphasizing that this biopharmaceutical risk evaluation is continuously facilitated by generated clinical data that allow for further evaluation of the in vivo relevance of the dissolution method and appropriate refinements as needed. Typically, a sponsor's strategy to understand and control biopharmaceutical risk can be described by two general approaches, as depicted in Fig. 2. These approaches are described in more detail below. Approach 1. The sponsor may determine that little additional PK evaluation may be needed, as processing and compositional controls are available to mitigate against any significant biopharmaceutical risk. In this case, in vivo studies using formulation variants would not be performed and manufacturing would continue within established ranges/parameters. For drug products comprising BCS classes 1 and 3 that exhibit rapid or very rapid dissolution (i.e., $\geq 85 \%$ dissolved in 30 or $15 \mathrm{~min}$, respectively), the dissolution criteria could be set accordingly. For other drug products, the dissolution method and specification criteria would be established to prevent significant variations vs. batches used in pivotal clinical trials and registration stability batches. As these batches would have been made under conditions that are representative of the final commercial product manufacturing process and the clinical batches would have been used to demonstrate clinical safety and efficacy, the dissolution specification would be derived from these batches and thus assure in vitro performance.

Additionally, if the dissolution specification and method are established with no additional PK studies of formulation variants, this may add further challenges for scale-up and post-approval changes; depending on the level of the change, these changes often require $f_{2}$ dissolution similarity (either in the QC method or in multi-pH media, and water) or, if those requirements are not met, require a bioequivalency study. Approach 1 may be at risk of generating specification criteria that are conservative, such that lots that fail the specification may not in fact have unacceptable PK performance. Thus, justification of potentially "wider" specifications based on enhanced in vivo product understanding as explained below may be more desirable.

Approach 2. In this scenario, the sponsor may utilize additional bioavailability evaluation in order to further explore the impact of formulation and/or process variability and dissolution differences on PK performance. For the PK study, ideally, formulation or process variants are chosen to provide a range of dissolution responses to support a deeper product understanding and wider manufacturing conditions.

The outcome of these PK studies can yield information meeting the following four scenarios: IVIVC Level A, IVIVC

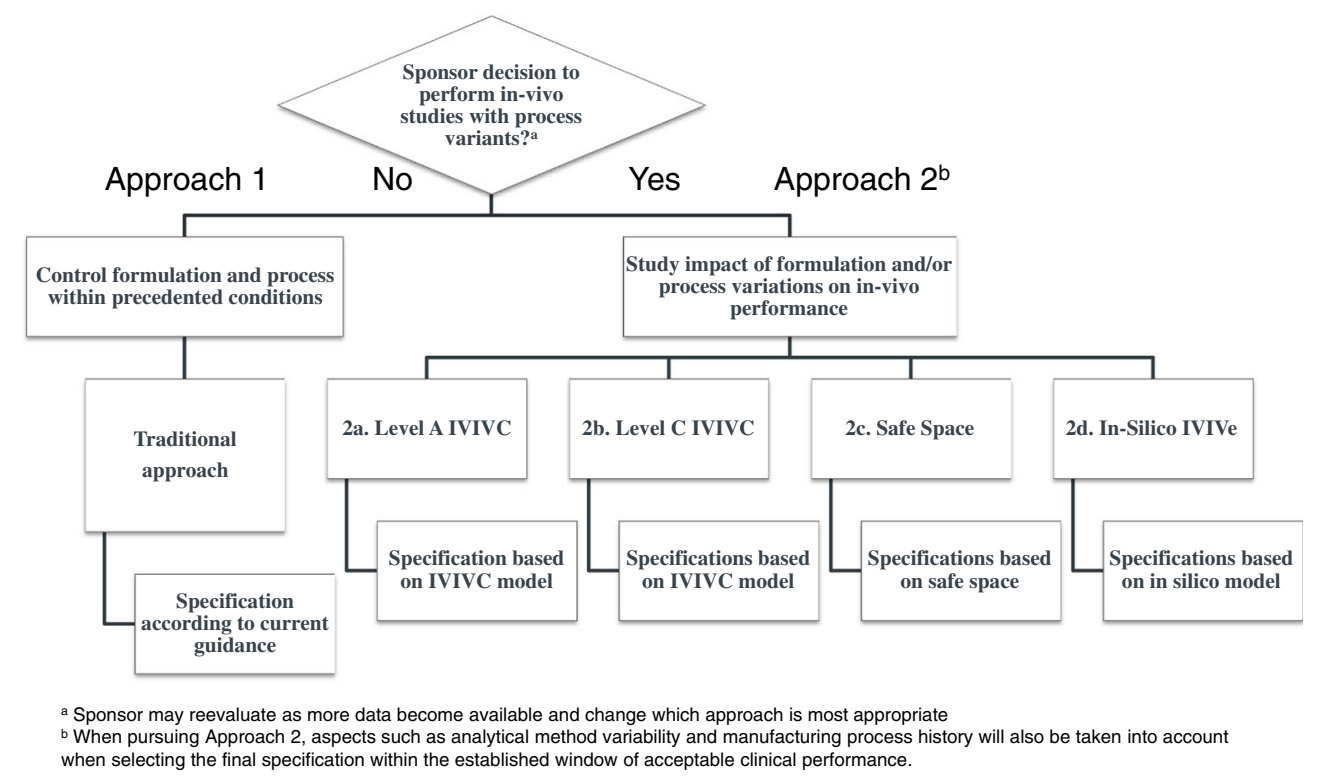

Fig. 2. Decision tree to develop clinically relevant specifications 
Level C, clinically established "safe space," and establishment of an in silico IVIVe.

1. An IVIVC Level A can be established if the dissolution method is predictive of in vivo PK performance. In this case, the method can be used to justify acceptance criteria and manufacturing conditions based on target PK parameters. An IVIVC Level A supersedes the $f_{2}$ similarity requirements and is acceptable from a regulatory perspective when applying for a biowaiver to support scale-up and post-approval changes.

2. An IVIVC Level $\mathrm{C}$ is established where only one or several PK parameters correlate with one or multiple dissolution time points. Therefore, the dissolution might not be predictive of the entire PK profile. For immediate release products, setting a dissolution specification for the QC method may be based on the lower range of the IVIVC model (10\% change in PK parameters relative to the target).

3. A clinically established "safe space" for dissolution can be established when formulation/process variants demonstrate acceptable PK performance, but the dissolution method can discriminate those variants. A dissolution specification for the QC method can be justified based on the limits of the release profiles used in the PK studies.

4. In silico IVIVe (in vitro/in vivo extrapolation) - in this scenario, the link between the in vitro dissolution and the observed pharmacokinetic response is established via the use of a physiologically based absorption/ pharmacokinetic model, and the model is used to identify dissolution profiles that are projected to ensure the desired clinical performance.

There are limited examples of Level A IVIVCs for IR products reported in the literature, and any level of IVIVC may not be expected for BCS 1/3 drugs or BCS 4 drugs with very low permeability as the dissolution rate in these cases is not the rate-limiting step to absorption. On the other hand, establishing an IVIVC Level C or a clinically established safe space may be realized for most drug products.

It should be noted that once the window of acceptable dissolution performance from a clinical perspective has been established under approach 2, other aspects of quality evaluation such as complete release, analytical and manufacturing process variability, and batch experience should also be taken into account when setting the final specification criteria within this range; in particular, it is desirable to avoid specifications set too tightly within the window of acceptable clinical performance that could result in the failure of clinically acceptable batches. A detailed discussion of the broader aspects of specification setting is beyond the scope of this manuscript; however, its impact is highlighted in the examples below.

In addition to setting clinically relevant dissolution specifications for finished product release as described above, similar concepts can be applied to setting acceptance criteria for formulation/material attributes. For example, the drug substance particle size distribution (PSD) often has an impact on drug product dissolution (Fig. 1). According to approach
1, understanding dissolution profiles of drug product made with API batches covering the expected PSD range of pivotal clinical batches would establish a way to ensure consistent product performance. Limits could be applied to the PSD range to ensure that significant changes in dissolution rate are not observed supported by in vitro dissolution testing with an appropriately discriminating method. Alternatively, following the logic presented in approach 2, drug product variants made with API batches covering the projected commercial API PSD range could be studied in vivo and compared to the PK performance of pivotal clinical studies. In either case, the PSD ranges can be justified; the in vitro dissolution method can be correlated with PSD and clinical data, and hence provide for clinically relevant specifications.

This specification setting strategy can be applied to other CMAs such as granule particle size, surface area, and lubricant levels, or drug substance properties such as API form changes and API disproportionation, or direct tablet properties such as hardness and porosity. Ultimately, this approach, which is based on sound product and process understanding, might lead developers to set specifications based on well-understood underlying CQAs, as opposed to depending on dissolution testing.

As mentioned above, during initial formulation and process evaluation and selection, dissolution may be conducted with a range of methods including non-traditional and traditional QC methods. Data from both types of methods can then be used to explore whether a direct linkage between dissolution and PK performance can be achieved (approach 2). With this information in hand, one can justify selection of appropriately discriminating method conditions (i.e., surfactant levels, $\mathrm{pH}$, agitation speeds) and acceptance criteria for a QC method. If the QC method shows similar clinical relevance compared to the more complex method, then only the QC method would be used for product release and to justify formulation and process changes. On the other hand, the QC method, which from a company's perspective should be globally acceptable, may not be capable to demonstrate acceptable PK performance of extreme process and formulation variants. In this case, the QC method conditions and acceptance criteria are set to assure adequate product quality control for products made under standard/routine manufacturing conditions. In addition, a sponsor may use the second, more complex dissolution specification that is indicative of acceptable clinical performance to support product quality investigations or specific post-approval changes. The reason for not using the complex dissolution method for routine product release could be based on lack of method robustness or concerns of global regulatory acceptance when using non-traditional, perhaps novel technology.

It is worth noting that while the discussion above focuses on responding to the outcome of the clinical studies, another important aspect of the CRS roadmap is to foster improved clinical study design to achieve this desired outcome. In particular, dissolution data, along with oral absorption/PBPK modeling, guide the rational design of PK studies to establish a likely safe zone or IVIVCs for the critical formulation/ process parameters. Following the completion of a PK study to link in vitro and in vivo performance, any previously established model(s) can be used (or further refined) to mechanistically understand the output by identifying the key 
contributing factors for absorption, and characterizing the interplay between formulation performance and physiological factors. The utility of this approach to establish CRS is briefly discussed later in this manuscript.

As more data become available, the sponsor may decide to change the approach as deemed necessary. For example, if the sponsor originally embarked on approach 1 but found that the appropriate control of one of the critical parameters could not be guaranteed in a commercial setting, the sponsor may elect to run additional PK studies to support the required range (approach 2). Similarly, if the sponsor originally embarked on a plan to develop an IVIVC but later assessed that the bioperformance risks are manageable through reasonably tight process controls, the sponsor might elect instead to accept the traditional approach of setting specifications according to approach 1 . Either of these should be considered acceptable.

\section{CASE STUDIES}

Two case studies are presented demonstrating outcomes $2 \mathrm{~b}$ and $2 \mathrm{c}$ as described in Fig. 2. In case study 1, a multiple Level C IVIVC was established. This relationship was used to support a clinically relevant specification and in-process control on tablet hardness. For case study 2, a pharmacokinetic safe space was established. This study also highlights the challenges of applying this approach currently in a global environment.

Case Study 1: Utilization of Dissolution Mechanism Understanding and Multiple Level C IVIVC to Inform Clinically Relevant Specifications for Suvorexant Solid Dispersion Tablets (30)

Suvorexant is an orexin receptor antagonist approved for the treatment of insomnia in the USA (Belsomra ${ }^{\mathrm{TM}}$ ). The active ingredient is a BCS class 2 compound formulated as an amorphous solid dispersion to enhance its inherently low crystalline solubility. Following the principles outlined under the CRS roadmap, during formulation development increased emphasis was placed on understanding the dissolution mechanism. Suvorexant tablets are manufactured via direct compression of a milled solid dispersion intermediate mixed with excipients. Therefore, the dissolution can be described as a two-step process: tablet erosion or disintegration followed by dissolution of the amorphous intermediate particles. During formulation development and scale-up, tablet erosion, and not API/solid dispersion intermediate dissolution, was demonstrated to be the rate-limiting step for overall dissolution of the dosage form. The CMA-controlling tablet erosion for the suvorexant formulation was found to be tablet solid

1 If $f_{2}$ was chosen to guide product development and to assess potential future risk with post-approval changes, one should consider selecting process variants that would fail the $f_{2}>50$ requirement so that those post-approval changes could be potentially justified provided acceptable bioperformance is demonstrated. fraction, which can be modulated by changes in tablet hardness and moisture.

Following the CRS decision tree (Fig. 2), a relative bioavailability study with four batches of tablets with varying hardness was executed to probe the impact of tablet erosion on in vivo performance. Tablet hardness was selected to bracket the dissolution of phase 3 supplies and to also result in sufficient dissolution difference ${ }^{1}$ between batches to enable development of correlations between dissolution and PK. Given that Level A IVIVCs are generally considered challenging for IR products, at the time of study execution the primary goal was the development of a multiple Level $\mathrm{C}$ IVIVC (outcome $2 b$ in the CRS roadmap).

The study outcome and the development of the multiple Level C IVIVC have been detailed in a previous publication (30). While tablet hardness did not impact the extent of absorption (AUC), modest differences in the rate of absorption $\left(C_{\max }\right)$ were observed. Figure 3 shows the target dissolution profile along with two sets of boundaries, those set according to the $f_{2}$ test and those set via the IVIVC. The target commercial manufacturing dissolution profile (black line) was based on the dissolution behavior observed with the phase 3 formulation. The $f_{2}$ bounds $(+/-10 \%)$ around that profile, which are typically used to assess dissolution similarity for post-approval changes, are represented by the dashed lines. The magenta lines represent the estimated bounds from the IVIVC model that would ensure bioequivalence as defined by changes in $C_{\max }$ within $\pm 10 \%$ relative to the target dissolution. It is apparent that applying strict $f_{2}$ criteria, implying a dissolution specification of approximately $Q=80 \%$ in $20 \mathrm{~min}$ (i.e., $\geq 85 \%$ dissolved in $20 \mathrm{~min}$ ), is too conservative and not reflective of the in vivo performance of the formulation. The understanding of the dissolution $v s . C_{\max }$ response led to clinically relevant dissolution bounds outside the $f_{2}$ range, while still ensuring consistent bioperformance of future batches.

Furthermore, as a link between formulation properties and dissolution is established, the IVIVC can be further utilized to inform manufacturing conditions/in-process controls. For the case of suvorexant tablets, the dissolution data were correlated to tablet tensile strength across potencies as described before (Fig. 4). This relationship was used to set an upper limit on tablet tensile strength and, through that parameter, tablet hardness. Tablet friability was used to set the lower limit. In this manner, this

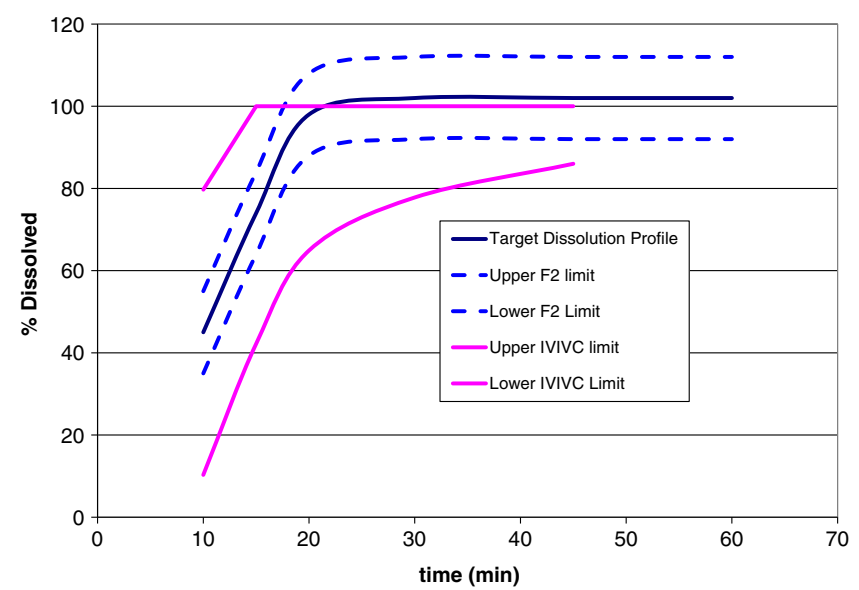

Fig. 3. Phase 3 "target" dissolution, corresponding $f_{2}$ range, and IVIVC-estimated bioequivalent dissolution space for suvorexant IR tablets 


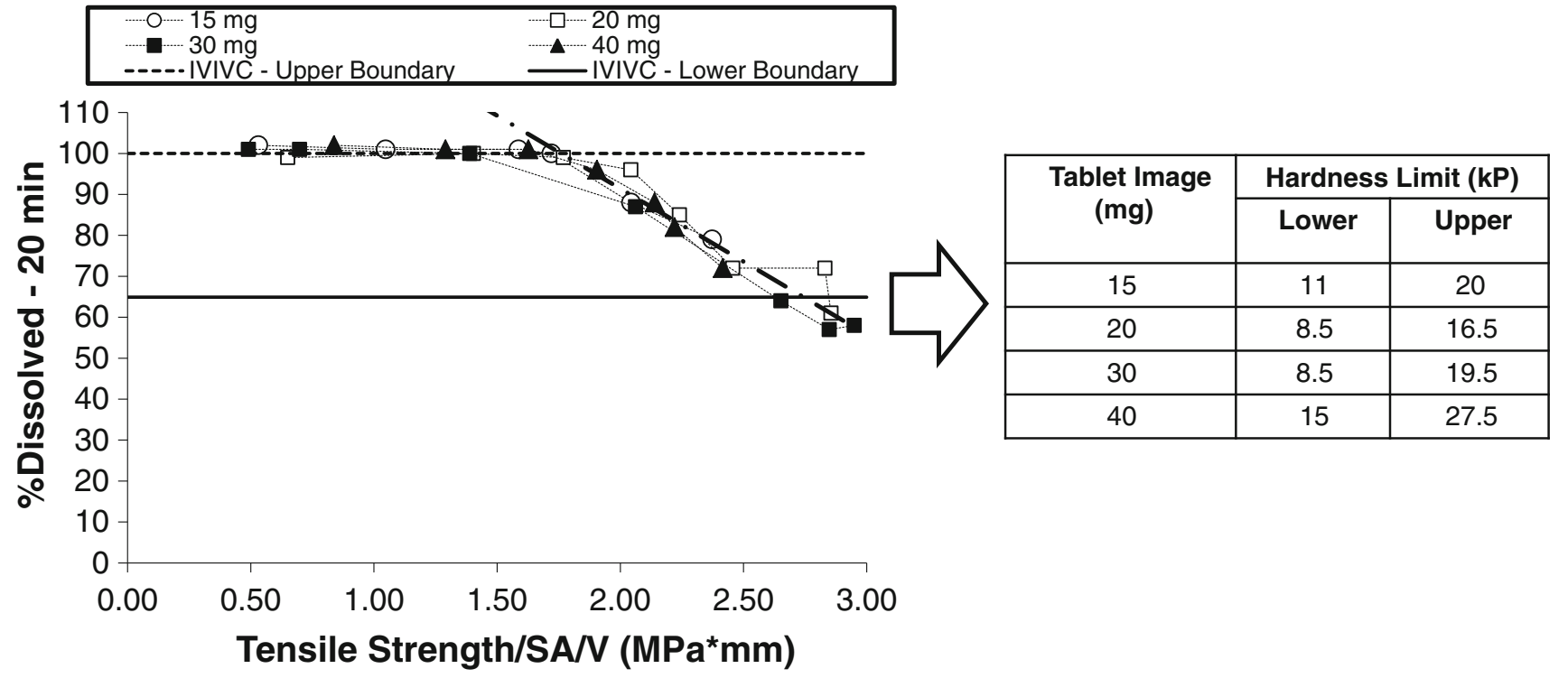

Fig. 4. Relationship between dissolution and tablet strength and translation to hardness limits for in-process control (30)

approach can contribute to the development of an effective control strategy where end-product testing (i.e., dissolution testing) serves as a final confirmation of the quality and required quality is built in at a more fundamental, and earlier, level.

In addition to informing the bioequivalent dissolution space for the dissolution specification (and subsequently hardness limits) for the suvorexant tablets, the IVIVC developed was also applied to project bioequivalence (BE) for different strength tablets (dose/weight multiples). Given the erosion mechanism, lower strengths (i.e., smaller tablets) dissolve faster, such that dissolution comparisons did not meet the $f_{2}$ criterion. Given that multiple strengths were considered at that time of development, demonstrating interchangeability for weight multiple tablets was desired for specific markets. To assess the bioequivalence for $2 \times 20 \mathrm{mg} \mathrm{vs}$. $1 \times 40 \mathrm{mg}$ and $2 \times 15 \mathrm{mg}$ vs. $1 \times 30 \mathrm{mg}$ tablets, the IVIVC model was applied. Based on the 20-min dissolution time point, the maximal differences projected between $2 \times 15 \mathrm{mg}$ and $1 \times 30 \mathrm{mg}$ tablets was $9.5 \%$ on $C_{\max }$ (projected differences for $2 \times 20 \mathrm{mg} v s$. $1 \times 40 \mathrm{mg}$ were smaller). The IVIVC projections were utilized internally to design and power a bioequivalence study to compare weight multiple formulations. As shown in Table I, bioequivalence criteria were met for both comparisons attempted (both AUC and $\left.C_{\max }\right)$ in line with the predictions of the IVIVC Level C model. The successful prediction of bioequivalence for these tablets can be also considered an external validation of the IVIVC model.

In summary, the establishment of a multiple Level C IVIVC for this product enabled a better understanding of the clinical (in this case pharmacokinetic) impact of process changes and dissolution. This increased product understanding is at the heart of a QbD strategy. The data from the IVIVC can be used to establish clinically relevant specifications and adequate in-process controls.

Case Study 2: Developing Clinically Relevant Specification for a BCS Class 4 Immediate Release Product-Establishing a Pharmacokinetic "Safe Space"

The second case study presented describes the development of a clinically relevant dissolution test and acceptance criterion for a BCS class 4 compound, formulated as an immediate release tablet. While the first case study resulted in an IVIVC, this example discusses the demonstration of a pharmacokinetic safe space to define the acceptable performance bounds of formulation changes.

Compound $\mathrm{X}$ is a BCS class 4 compound with poor solubility $(<10 \mu \mathrm{g} / \mathrm{mL})$ in buffers within the physiological $\mathrm{pH}$ range. However, the compound demonstrates solubility of $0.5 \mathrm{mg} / \mathrm{mL}$ in human intestinal fluid. The physicochemical properties of compound $\mathrm{X}$ are summarized in Table II.

The first step in establishing a clinically relevant dissolution test for compound $\mathrm{X}$ was to identify the most relevant risks to in vivo dissolution. The product is manufactured by a standard wet granulation process, and the formulation includes a superdisintegrant to facilitate rapid disintegration and dissolution. As API particle size and crystalline form had already been fixed in an area of low risk, Quality Risk

Table I. Application of Multiple Level C IVIVC to Estimation of Bioequivalence for Different Strength Suvorexant Tablets and Comparison to BE Study Outcome

\begin{tabular}{|c|c|c|c|c|}
\hline Tablet strength & $\mathrm{AUC}_{0-\mathrm{t}}$ prediction & $\mathrm{AUC}_{0-\mathrm{t}} \mathrm{BE}$ results & $\begin{array}{l}C_{\max } \text { prediction based on IVIVC } \\
\text { ( } 20 \text { min time point) }\end{array}$ & $C_{\max }$ results \\
\hline $\begin{array}{l}2 \times 20 \mathrm{mg} \mathrm{vs} . \\
1 \times 40 \mathrm{mg}\end{array}$ & $\begin{array}{l}\text { IVIVC not applied. AUC not } \\
\text { sensitive to dissolution }\end{array}$ & $102.52 \%(99.09-106.07)$ & $105.3 \%$ & $96.58 \%(90.96-102.55 \%)$ \\
\hline $\begin{array}{l}2 \times 15 \mathrm{mg} v s . \\
1 \times 30 \mathrm{mg}\end{array}$ & $\begin{array}{l}\text { IVIVC not applied. AUC not } \\
\text { sensitive to dissolution }\end{array}$ & $99.71 \%(96.66-102.75 \%)$ & $109.5 \%$ & $108.74 \%(101.10-116.95 \%)$ \\
\hline
\end{tabular}


Table II. Physicochemical Properties of Compound X

\begin{tabular}{ll}
\hline Solubility in & $<10 \mu \mathrm{g} / \mathrm{mL}$ across the physiological \\
aqueous buffers & $\mathrm{pH}$ range
\end{tabular}

Solubility in

biorelevant media

Permeability

$0.5 \mathrm{mg} / \mathrm{mL}$ in human intestinal fluid

Moderate permeability in Caco-2 cells; absolute bioavailability $36 \%$

Assessment (QRA) had identified granulation parameters and disintegration time (e.g., due to a change in the performance of the binder or superdisintegrant) as the highest risks. Process and formulation variants were then produced which investigated ranges for these risks, as described in Table III. The critical mechanisms of overgranulation and over-compression were combined to create larger changes in dissolution performance.

Dissolution tests were conducted to assess the potential impact of these highest risk failure mechanisms. The poor aqueous solubility of compound $\mathrm{X}$ meant that aqueous buffers at physiologically relevant $\mathrm{pHs}$ were unsuitable for use as dissolution media, as less than $10 \%$ of the dose would dissolve in a 900-mL volume. Surfactant-containing media were therefore investigated. Tablet performance was also assessed in Fasted State Simulated Intestinal Fluid (FaSSIF), as a known biorelevant medium, for comparison. The process and formulation variants showed slower dissolution in all of the media assessed (Fig. 5); different degrees of discrimination were observed, but the rank order of profiles was the same.

The next step was to determine the in vivo impact of the in vitro differences observed. As compound $\mathrm{X}$ is characterized

Table III. Tablet Variants Incorporating Highest Risks to In Vivo Dissolution

\begin{tabular}{ll}
\hline Description & Dissolution Risks \\
& Addressed \\
& from QRA
\end{tabular}

Variant A Process variant: overgranulated and over-compressed

Variant B Process variant: overgranulated (extreme) and over-compressed, only large $(>1 \mathrm{~mm})$ particles used for compression

Variant C Formulation variant: increase the amount of binder and reduce the amount of disintegrant to slow tablet disintegration as BCS class 4, a clinical relative bioavailability study was conducted to evaluate the in vivo impact of the dissolution differences. A study was performed (in healthy volunteers), comparing exposures from the tablet variants to the standard clinical tablet, as well as an oral solution.

All of the tablet variants were found to be bioequivalent to the standard tablet (Table IV). Figure 6 shows the geometric mean pharmacokinetic profiles obtained. Although the profiles appear to show some differentiation, examination of individual PK data demonstrated that there was no consistent rank order for the tablet variants vs. the standard tablet between individual subjects in the study. The oral solution showed a slightly higher $C_{\max }$ than the tablets.

These data were then used in conjunction with the discriminating dissolution methods to allow a specification to be set that assures in vivo performance. The clinical study had established a range of in vitro dissolution profiles within which bioequivalence was assured, enabling a safe space for dissolution performance to be defined (9). The study had shown that the in vitro dissolution methods in Fig. 5 all discriminate for changes in in vitro performance which do not impact in vivo performance, so any of the dissolution methods would be suitable for use as a clinically relevant QC method using the safe space approach. A release profile anywhere within the identified region of bioequivalence in any one of these tests would be suitable to assure clinical quality. In this instance, the project team selected one of the surfactant-based media with a similar degree of discrimination to FaSSIF as a QC release test. Preliminary discussions with FDA regarding appropriate discriminating power indicated a preference for a method with more discrimination for the initial rate phase than was seen with surfactant 1 .

The clinically relevant dissolution method and specification were used during subsequent product and process development to assess the impact of lower risk variables that had not been evaluated in the clinical study. The clinically relevant dissolution test and specification are therefore an integral part of the wider control strategy, ensuring that every tablet produced during commercial manufacture will be bioequivalent to those dosed in the pivotal safety and efficacy studies.

At the time of filing the marketing applications, a dissolution specification of $Q=70 \%$ (i.e., $\geq 75 \%$ dissolved) at 45 min was proposed. This was accepted by all agencies except US FDA, who requested a $Q=80 \%$ (i.e., $\geq 85 \%$ dissolved) specification, to provide assurance of complete release. Following discussion with the Agency, a two-point specification of $Q=70 \%$ at $45 \mathrm{~min}$ and $Q=80 \%$ at 60 min was agreed; the sponsor stated their intention to review the need for a two-point specification when a significant body of commercial batch data was available.

pt? $>$ As more experience of variability during routine commercial manufacture was gained, statistical modeling of manufacturing process performance identified a risk that clinically acceptable batches from within the defined safe space for compound $\mathrm{X}$ could potentially fail specification. This was a particular risk for the $Q=80 \%$ in 60 min US specification, which was set relatively tight for such a highly discriminating in vitro test. Based on these data, further dialog was sought with FDA, and the US specification was successfully re-negotiated to $Q=75 \%$ at $60 \mathrm{~min}$, significantly reducing the risk of failing clinically acceptable batches. This highlights a key value of a clinically relevant dissolution test and associated pharmacokinetic safe space, as developed for compound $\mathrm{X}$. This also highlights the importance of 


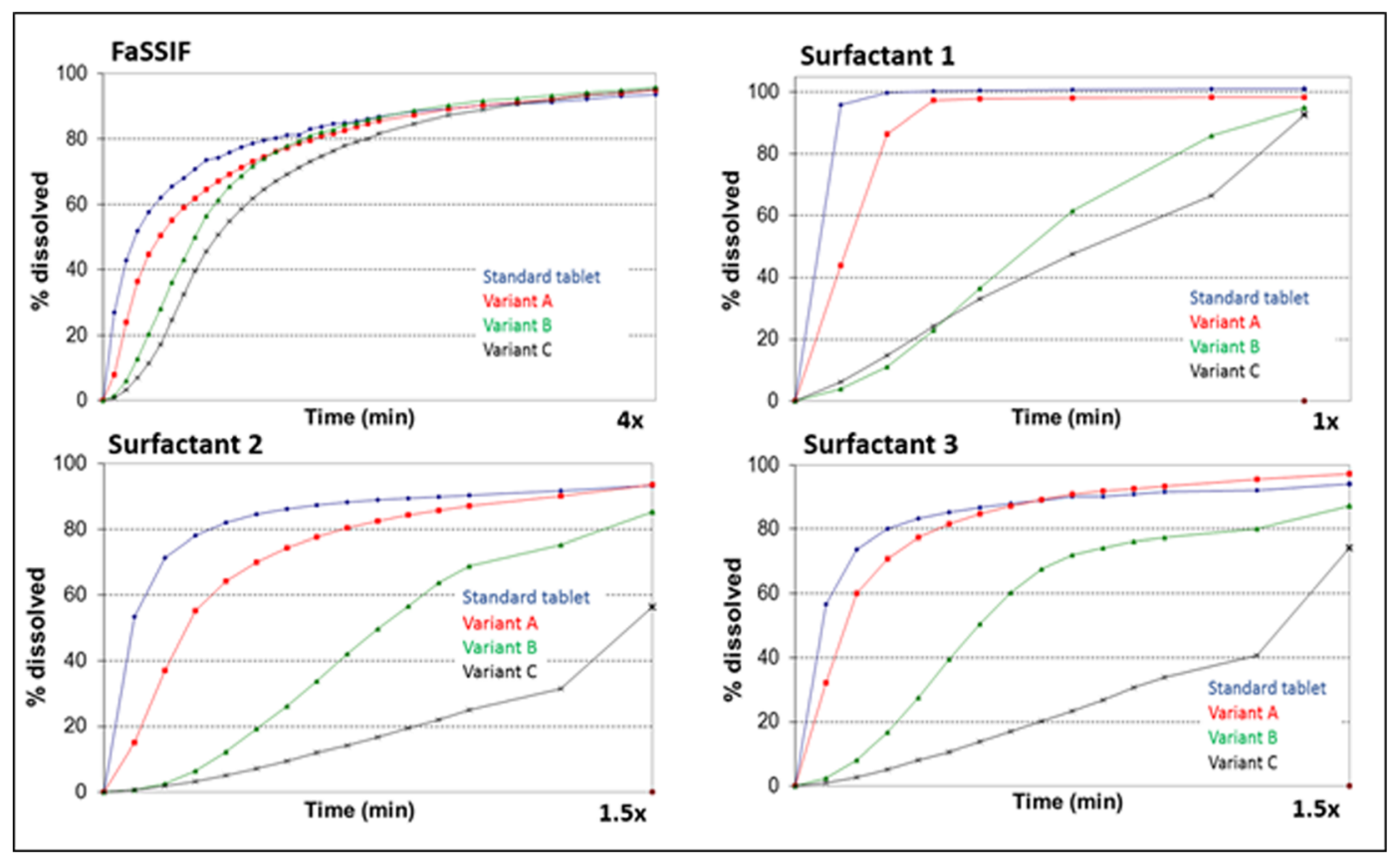

Fig. 5. Dissolution of the tablet variants in FaSSIF and three surfactant-containing media

considering the constraints of a commercial manufacturing environment in setting the final specification.

During the development of the above referenced IR tablet, two clinically relevant dissolution methods were identified as potentially being suitable for routine batch release of this product. One method, using a pH 1.2 aqueous buffer, only discriminated for the most slowly dissolving tablet variant. The second method used a medium containing $0.5 \%$ surfactant and was able to discriminate between all of the variants dosed in the study, similar in discriminatory power to the biorelevant FaSSIF medium. In the marketing applications, the sponsor proposed the surfactant method with an associated single-point specification. Differing feedback was obtained from regulatory authorities globally on the suitability of the method and the proposed specification; this has been described by Timko et al. (31). In brief, US FDA agreed that the surfactant method was suitable for use as a pharmaceutical development tool, but noted that it could be overly sensitive for changes in product performance that had no impact clinically. FDA requested that the $\mathrm{pH} 1.2$ method be used for batch release, as this was considered to be more biorelevant, while other regulatory authorities, including EMA and Health Canada, accepted the originally proposed surfactant-based method.

Both methods and acceptance criteria can be considered clinically relevant, as they would pass clinically acceptable

Table IV. Comparison of Exposures from the Tablet Variants Compared to the Standard Tablet, According to Standard Bioequivalence Criteria

\begin{tabular}{lll}
\hline & $\begin{array}{l}\text { AUC } G_{\text {mean }} \\
\text { ratio }(90 \% \text { CIs })\end{array}$ & $\begin{array}{l}C_{\text {max }} G_{\text {mean }} \\
\text { ratio }(90 \% \text { CIs })\end{array}$ \\
\hline Variant A/standard tablet & $0.97(0.90,1.05)$ & $1.05(0.95,1.16)$ \\
Variant B/standard tablet & $1.02(0.94,1.10)$ & $1.04(0.94,1.15)$ \\
Variant C/standard tablet & $0.97(0.89,1.05)$ & $0.91(0.83,1.00)$ \\
\hline
\end{tabular}

batches and fail batches with unacceptable bioperformance. This example highlights that there can be differing QC dissolution testing strategies based on country-specific regulatory expectations. In this case, the divergence in regulatory expectations have resulted in a more complex global supply chain, with different QC dissolution tests and specifications used to release batches in different regions. Taken together, this example and the case study described above illustrate that this is an evolving area of regulatory and pharmaceutical science, which warrants further investigation and discussion.

\section{SELECTION OF APPROPRIATE TOOLS FOR ESTABLISHING CRS}

The CRS principles outlined in this manuscript are in line with the FDA's dissolution guidance from 1997 for IR products and the recently proposed Biopharmaceutics Risk Assessment Roadmap (BioRAM) which calls for the use of preclinical tools and clinical studies to gradually develop the product understanding in order to maximize patient benefit. As discussed in the corresponding BioRAM manuscript (32), generalized delivery scenarios can be assigned to help identify a drug product's critical performance attributes. The same concepts could be applied to selecting the appropriate tools for generation of a clinically relevant dissolution specification. For example, for MR products, it is generally accepted that the entire time course of the plasma concentration profile is important in the majority of cases; thus, as reflected in current regulatory guidances, Level A IVIVCs are preferred. In contrast, for IR products where efficacy/safety is typically linked to a summary pharmacokinetic parameter (e.g., AUC, $C_{\max }, C_{\text {trough }}$ ), Level C IVIVCs (ideally multiple Level C IVIVCs) may be adequate to ensure product performance. The individual drug efficacy/safety characteristics and established margins would need to be taken into account. For certain products and indications, one may be just looking for a change in $C_{\max }$ with little effect of formulation variants on AUC. If $C_{\max }$ is important for efficacy (e.g., an analgesic drug), CRS should focus on characterizing that 


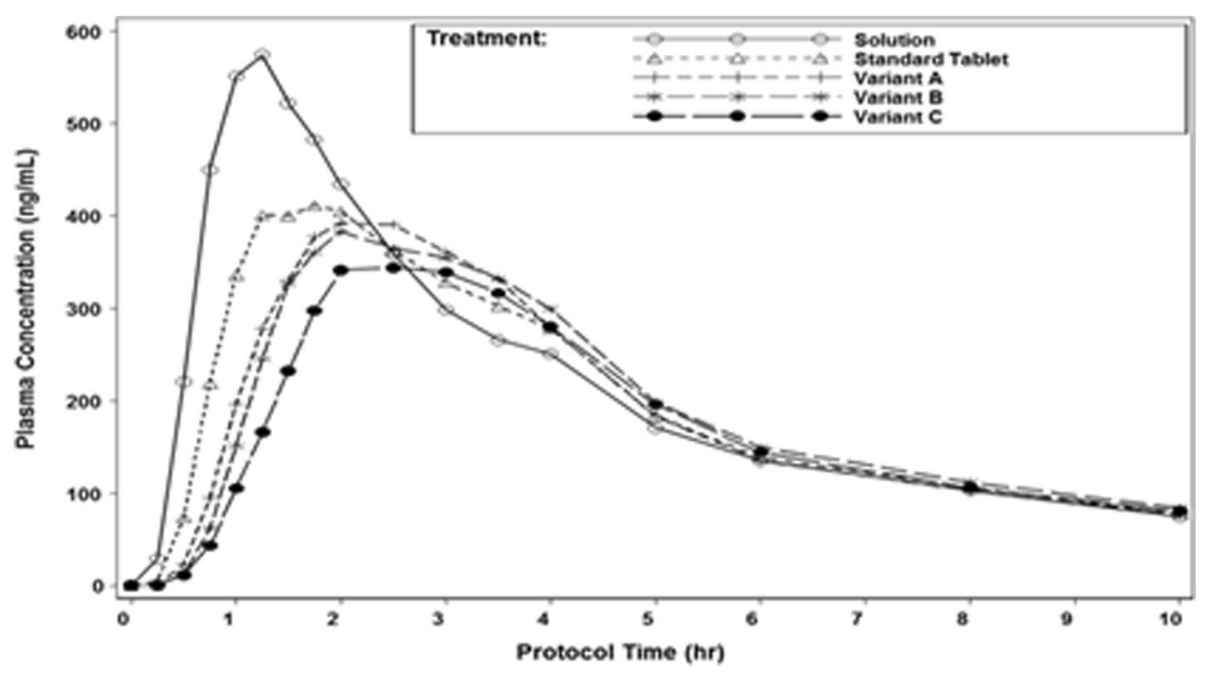

Fig. 6. Plasma concentration time profiles for the standard tablet, oral solution, and tablet variant

relationship to formulation parameters. On the other hand, if the drug is used for an indication where AUC or $C_{\text {trough }}$ is important (e.g., anti-viral), the safe space concept applied to AUC may be the best surrogate to establish a product specification.

In recent years, utilization of physiologically based oral absorption/pharmacokinetic models related to biopharmaceutics and formulation performance has been significantly increasing within the industry. Several publications both from industry and from regulatory agencies have highlighted the potential use of such models, understanding formulation critical attributes and dissolution performance. For example, Zhang et al. demonstrated how oral absorption/PBPK modeling could be used to understand the in vivo performance of generic IR and XR carbamezepine formulations and identify the important characteristics in the release mechanisms that should be reflected in a dissolution test (33). More recently, Mitra et al. described the use of models to assess the impact of dissolution differences observed between batches manufactured at different sites for etoricoxib (34). The simulations showed that the dissolution differences (outside $f_{2}$ bounds) observed at $\mathrm{pH} 4.5$ and 6.8 during multimedia testing were not clinically relevant, while the dissolution at $\mathrm{pH} 1.2\left(f_{2}>50\right)$ would be a better predictor of clinical performance. Results from a bioequivalence study confirmed the outcome of the simulations. Finally, Pepin et al. recently published their experience with utilizing absorption/PBPK modeling to justify drug product dissolution and drug substance particle size specification for Zurampic® (lesinurad) IR tablets (35). While a detailed review of the development and application of such physiologically based oral absorption/pharmacokinetic models is beyond the scope of this manuscript, the three examples highlighted demonstrate the potential utility of modeling in conjunction with the more traditional IVIVC approaches and the safe space concept discussed in the case studies to develop CRS.

\section{EXECUTION OF CRS DURING CLINICAL DEVELOPMENT}

The approaches the sponsor can take to demonstrate clinical relevance of the dissolution specification have been discussed broadly in presentations given by the FDA and industry. Furthermore, an aspirational goal would be to enable product release without end-product testing, insofar as process controls would guarantee product quality. To achieve this goal, deep product knowledge, including formulation components (excipients, API), process, and stability, is required, as well as the tools (methods) to probe whether formulation and process changes impact product quality or not.

Product formulation and process understanding is evolving throughout clinical development, offering a range of opportunities for establishing CRS during drug product development and post-market approval. Early in development, comprehensive studies of formulation prototypes prior to and during phase 1 are generally not performed, as the formulations used are usually exploratory, and these studies may not provide information that is pivotal to the final product. Nevertheless, in-human data generated this early in development may provide valuable bioperformance knowledge that may guide formulation platform selection and better understanding of API attributes.

When compounds are entering clinical phases 2 and 3, performing PK experiments with formulation variants as part of enhanced product understanding becomes more relevant. It is typically at this stage when the potential need to perform PK studies to support process operating ranges becomes clear. In addition, the designs of the actual clinical studies, with respect to the selection of which formulation/process variants to evaluate, are determined. This is an outcome of the ongoing risk assessments. At this stage of product development, a fundamental understanding of the ratelimiting step in the dissolution mechanism should be available. This allows fine tuning method conditions for traditional dissolution experiments, or it might, in fact, highlight the need to develop additional methodologies which correlate one or more formulation material attributes with the observed PK performance. Furthermore, at this stage, M\&S may provide significant opportunity on its own or as supplement to PK experimentation to develop CRS.

During phase 3 development, the formulation and process is usually "locked" which means that the materials to support pivotal clinical studies have been released and registration stability with material made at the appropriate scale may have already started. For most programs entering phase 3 clinical development, the dissolution method 
conditions used for clinical batch release, registration stability, and additional scale-up development are in place. However, acceptance criteria for the QC dissolution method ${ }^{2}$ are usually finalized after all relevant stability and clinical batch data have been evaluated. Despite the advanced stage of the program, targeted PK studies with process and formulation variants could be conducted to further support clinically relevant specification setting. The scope of such studies may be narrower than the PK studies conducted earlier in development. In practice though, performing additional PK studies is often only pursued if risks cannot be mitigated without significant changes in formulation and/or process that could potentially question the relevance of the data from phase 3 clinical efficacy and safety studies. If the additional clinical PK studies support changes to the dissolution method and/or changes in the formulation/process conditions, this information could serve as additional justification of the proposed commercial control strategy.

\section{CRS AND POST-APPROVAL CHANGES}

Current discussions about CRS dissolution specifications focus heavily on product development, but the concept is equally applicable after product approval and for generic product development. As clinically relevant dissolution specifications are more likely to identify a potential bioperformance risk, this should be seen as an opportunity for regulators and industry to continue working together toward global harmonization on the use of CRS for post-approval changes. Specifically, this means that a clinically relevant dissolution specification should be acceptable to support post-approval changes.

Conceptually, the principles outlined in the extended release IVIVC guidance (36) could be applied to immediate release dosage forms and CRS in general. However, for most IR products, the development of a Level A IVIVC is highly unlikely even if extreme process and formulation variants were studied in vivo. Based on current regulatory guidances, only a Level A IVIVC would enable the use of the IVIVC predictive model for post-approval changes and biowaivers. The suvorexant example (case study 1 which follows approach $2 b$ in the Fig. 2 CRS roadmap), however, demonstrates that a multiple Level C IVIVC can also deliver an appropriate model to accurately predict the $C_{\max }$ and AUC of a formulation based on dissolution performance. Therefore, depending on the drug product's safety and efficacy (PK/PD), a Level C IVIVC, potentially strengthened by oral absorption/PBPK modeling, should be suitable to appropriately predict bioequivalence for post-approval changes (e.g., SUPAC Level 2 formulation changes) and/or biowaivers. Even in cases of a demonstrated safe space (e.g., case study 2 which follows approach $2 \mathrm{c}$ ), it should be feasible to apply the clinical experience for assessing SUPAC Level 1 and 2 formulation changes. In such a case, the clinically relevant dissolution method, rather than a traditional (non-CRS disso method) or multimedia dissolution profiles which both

2 Dissolution method conditions may still evolve even in phase 3 development; however, changes during, for example, registration stability studies, or to compare individual clinical batches used in pivotal safety and efficacy studies need to be carefully justified. require meeting the $f_{2} \geq 50$ requirements and are currently mandated by SUPAC IR, should be used to evaluate if the changes have an impact on bioperformance.

For products developed and commercialized following approach 1 (Fig. 2), the current SUPAC guidance seems appropriate. In this case, if the QRM has been performed rigorously, SUPAC formulation changes up to level 2 and process changes are unlikely to have an impact on bioperformance.

\section{RECOMMENDATIONS AND CONCLUSIONS}

Dissolution testing remains an important tool for product development and routine product release. Drug product dissolution comparisons, whether through the use of statistical approaches such as $f_{2}$ or based on IVIVC type studies, are key for evaluating if the product meets the desired bioperformance criteria, thus supporting process development and post-approval changes. The two case studies presented in this manuscript illustrate the potential to justify dissolution method conditions and acceptance criteria that are outside the current regulatory expectation. Given the aspiration to continuously improve the fundamental science that underpins the discriminating power of the dissolution method, as well as the goal of maximizing understanding of the manufacturing process, establishing clinically relevant dissolution specifications, based on process understanding coupled with PK data, appears to be a best practice.

The following recommendations reflect the current position of pharmaceutical companies represented by IQ on clinically relevant dissolution specification:

1. For drug products comprising BCS class 1 and 3 compounds that exhibit rapid or very rapid dissolution (i.e., $\geq 85 \%$ dissolved in 30 or $15 \mathrm{~min}$, respectively, in all three media, $\mathrm{pH} 1.2,4.5$, and 6.8), this is considered a clinically relevant specification and should be sufficient to support scale-up and post-approval changes without the need to provide dissolution data in additional media for certain changes (e.g., water and $\mathrm{pH}$ 7.5) or demonstrating similarity of dissolution profiles for BCS 1 compounds as long as test and reference product dissolve rapidly.

2. For drug products comprising BCS classes 2 and 4, or BCS class 1 and 3 compounds that do not meet the criteria of rapid or very rapid dissolution, then the current guidance on dissolution specifications and post-approval changes should be followed (approach 1), unless:

3. The sponsor performed studies that evaluated formulation variants in vivo, in which case the specification should be set accordingly (approach 2). In addition, this same methodology and specification should be considered sufficient to support the dissolution aspects of scale-up and post-approval changes.

\section{ACKNOWLEDGEMENTS}

The authors would like to thank the many reviewers from the IQ member companies that contributed to this manuscript. 
Open Access This article is distributed under the terms of the Creative Commons Attribution 4.0 International License (http://creativecommons.org/licenses/by/4.0/), which permits unrestricted use, distribution, and reproduction in any medium, provided you give appropriate credit to the original author(s) and the source, provide a link to the Creative Commons license, and indicate if changes were made.

\section{REFERENCES}

1. U.S. Department of Health and Human Services - Food and Drug Administration, Center for Drug Evaluation and Research (CDER). Guidance for industry-dissolution testing of immediate release solid oral dosage forms. 1997.

2. Woodcock J. The concept of pharmaceutical quality. American Pharmaceutical Review. 2004;7(6).

3. International Conference on Harmonization of Technical Requirements for Registration of Pharmaceuticals for Human use (ICH). ICH Harmonized Tripartite Guideline: Pharmaceutical Development, ICH Q8 (R2); 2009.

4. International Conference on Harmonization of Technical Requirements for Registration of Pharmaceuticals for Human use (ICH). ICH Harmonized Tripartite Guideline: Quality Risk Management, ICH Q9; 2005.

5. International Conference on Harmonization of Technical Requirements for Registration of Pharmaceuticals for Human use (ICH). ICH Harmonized Tripartite Guideline: Pharmaceutical Quality System, ICH Q10; 2005.

6. Yu LX. Pharmaceutical quality by design: product and process development, understanding, and control. Pharm Res. 2008;25(4):781-91.

7. Nasr MM. Implementation of quality by design $(\mathrm{QbD})$ — current perspectives on opportunities and challenges. FDA Pharmaceutical Science and Clinical Pharmacology Advisory Committee; 2011.

8. Yu LX, Amidon G, Khan MA, Hoag SW, Polli J, Raju GK, et al. Understanding pharmaceutical quality by design. AAPS J. 2014;16(4):771-83.

9. Dickinson PA, Lee WW, Stott PW, Townsend AI, Smart JP, Ghahramani P, et al. Clinical relevance of dissolution testing in quality by design. AAPS J. 2008;10(2):380-90.

10. Selen A, Cruanes MT, Mullertz A, Dickinson PA, Cook JA, Polli JE, et al. Meeting report: applied biopharmaceutics and quality by design for dissolution/release specification setting: product quality for patient benefit. AAPS J. 2010;12(3):465-72.

11. Clinically LR. Relevant specifications (CRS): a regulatory perspective. PQRI: Conference on Evolving Product Quality; Bethesda; 2014.

12. Pope MS. Specifications for QbD-containing applications. AAPS workshop: facilitating oral product development and reducing regulatory burden through novel approaches to assess bioavailability/bioequivalence. Washington; 2011.

13. Suarez-Sharp S. Case studies: models for establishing clinically relevant drug specifications. AAPS Annual Meeting and Exposition. Washington; 2011.

14. Suarez-Sharp S. Establishing clinically relevant drug specifications: FDA perspective. AAPS Annual Meeting and Exposition. Chicago; 2012.

15. Norris K, Diaz DA, Ganapathy M, Gold L, Zhang L. Moving toward clinically relevant dissolution specifications. AAPS News Magazine. 2016.

16. U.S. Department of Health and Human Services-Food and Drug Administration, Center for Drug Evaluation and Research (CDER). Guidance for industry: Immediate Release Solid Oral Dosage Forms: Scale-Up and Postapproval Changes: Chemistry, Manufacturing, and Controls; In Vitro Dissolution Testing and In Vivo Bioequivalence Documentation. 1995.

17. U.S. Department of Health and Human Services-Food and Drug Administration, Center for Drug Evaluation and Research (CDER). Guidance for industry: SUPAC-MR: Modified Release
Solid Oral Dosage Forms : Scale-Up and Postapproval Changes: Chemistry, Manufacturing, and Controls; In Vitro Dissolution Testing and In Vivo Bioequivalence Documentation. 1997.

18. U.S. Department of Health and Human Services-Food and Drug Administration, Center for Drug Evaluation and Research (CDER). Dissolution Testing and Specification Criteria for Immediate-Release Solid Oral Dosage Forms Containing Biopharmaceutics Classification System Class 1 and 3 Drugs-Guidance for Industry—Draft Guidance. 2015.

19. Cook JA. Development strategies for IVIVC in an industrial environment. Biopharm Drug Dispos. 2012;33(7):349-53.

20. Marroum PJ. Clinically relevant dissolution methods and specifications. Am Pharm Rev. 2012;15(1):36-41.

21. Marroum P. History and evolution of the dissolution test. Dissolution Technologies. 2014;11-6.

22. Reppas C, Friedel HD, Barker AR, Buhse LF, Cecil TL, Keitel S, et al. Biorelevant in vitro performance testing of orally administered dosage forms-workshop report. Pharm Res. 2014;31(7):1867-76.

23. Nicolaides E, Symillides M, Dressman JB, Reppas C. Biorelevant dissolution testing to predict the plasma profile of lipophilic drugs after oral administration. Pharm Res. 2001;18(3):380-8.

24. Trasi NS, Purohit HS, Wen H, Sun DD, Taylor LS. Non-sink dissolution behavior and solubility limit of commercial tacrolimus amorphous formulations. J Pharm Sci. 2017;106(1):264-72.

25. Sun DD, Wen H, Taylor LS. Non-sink dissolution conditions for predicting product quality and In Vivo performance of supersaturating drug delivery systems. J Pharm Sci. 2016;105(9):2477-88.

26. Takeuchi S, Tsume Y, Amidon GE, Amidon GL. Evaluation of a three compartment in vitro gastrointestinal simulator dissolution apparatus to predict in vivo dissolution. J Pharm Sci. 2014;103(11):3416-22.

27. Jones HM, Chen Y, Gibson C, Heimbach T, Parrott N, Peters SA, et al. Physiologically based pharmacokinetic modeling in drug discovery and development: a pharmaceutical industry perspective. Clin Pharmacol Ther. 2015;97(3):247-62.

28. Kesisoglou F, Chung J, van Asperen J, Heimbach T. Physiologically based absorption modeling to impact biopharmaceutics and formulation strategies in drug development-industry case studies. J Pharm Sci. 2016;105(9):2723-34.

29. U.S. Department of Health and Human Services-Food and Drug Administration, Center for Drug Evaluation and Research (CDER). Waiver of In Vivo Bioavailability and Bioequivalence Studies for Immediate-Release Solid Oral Dosage Forms Based on a Biopharmaceutics Classification System, Rev 1. Guidance for Industry - Draft Guidance. 2015.

30. Kesisoglou F, Hermans A, Neu C, Yee KL, Palcza J, Miller J. Development of in vitro-in vivo correlation for amorphous solid dispersion immediate-release suvorexant tablets and application to clinically relevant dissolution specifications and in-process controls. J Pharm Sci. 2015;104(9):2913-22.

31. Timko RJ, Dickinson PA, Holt DJ, Montgomery FJ, Reynolds GK, Stott PW, et al. Quality-by-design: differing dissolution philosophies. AAPS Annual Meeting. San Antonio 2013.

32. Selen A, Dickinson PA, Mullertz A, Crison JR, Mistry HB, Cruanes MT, et al. The biopharmaceutics risk assessment roadmap for optimizing clinical drug product performance. J Pharm Sci. 2014;103(11):3377-97.

33. Zhang X, Lionberger RA, Davit BM, Yu LX. Utility of physiologically based absorption modeling in implementing quality by design in drug development. AAPS J. 2011;13(1):59-71.

34. Mitra A, Kesisoglou F, Dogterom P. Application of absorption modeling to predict bioequivalence outcome of two batches of etoricoxib tablets. AAPS PharmSciTech. 2015;16(1):76-84.

35. Pepin XJ, Flanagan TR, Holt DJ, Eidelman A, Treacy D, Rowlings CE. Justification of drug product dissolution rate and drug substance particle size specifications based on absorption PBPK modeling for Lesinurad immediate release tablets. Mol Pharm. 2016;13(9):3256-69.

36. U.S. Department of Health and Human Services-Food and Drug Administration, Center for Drug Evaluation and Research (CDER). Guidance for industry: Extended Release Oral Dosage Forms: Development, Evaluation, and Application of In Vitro/In Vivo Correlations. 1997. 